\title{
Efetividade do processo de comunicação com base na abordagem do comportamento informacional: o caso de um organismo internacional da área da saúde pública sediado no Brasil
}

\author{
Luciana de Deus Chagas \\ Mestre em ciência da informação \\ E-mail: luchagas28@yahoo.com.br \\ Sely de Souza Costa \\ PhD em ciência da informação \\ E-mail:selmar@unb.br
}

\section{RESUMO}

Aborda-se a efetividade do processo de comunicação organizacional do qual fazem parte 0 sítio web da Unidade Técnica de Sistemas e Serviços de Saúde da Organização Pan-Americana da Saúde (HSD/OPAS). A abordagem adotada fundamenta-se nos padrões de comportamento informacional de usuários do sítio da HSD/OPAS como insumo para o seu delineamento, a partir da noção de sistemas de informação apoiados em tecnologia. Utiliza-se a Metodologia de Sistemas Flexíveis (MSF), de Peter Checkland, com enfoque sistêmico e fenomenológico na identificação de ações que podem agilizar o processo de comunicação estudado, levando em conta os padrões de comportamento informacional de seus atores. Conclui-se que a efetividade do processo de comunicação entre gestores do setor de saúde pública e 0 sítio web da OPAS pode resultar da inserção dos padrões de comportamento informacional de gestores como subsídio ao delineamento do sistema de informação disponível no sítio web da HSD.

\section{PALAVRAS-CHAVE}

Comunicação organizacional. Gestão da saúde pública. Padrões de comportamento informacional. Processo de comunicação. Sistemas de informação.

\section{Effectiveness of the communication process based on the approach of information behaviour: the case of an international organism of public health located in Brazil}

\begin{abstract}
This study focuses on the effectiveness of a communication process comprised by the web site of the Technical Unit of Health Systems and Services of the PanAmerican Health Organization (HSD/PAHO) and managers of the public health sector. It is based on the concepts of information behaviour patterns of users and information systems supported by technology. The study adopted the Soft Systems Methodology (SSM), presented by Peter Checkland, which is based on the systemic thinking and phenomenological approaches. These approaches have been adopted in the proposal of actions that can contribute to making the communication process between sender and receptors more effective. The conclusion is that, based on the theoretical principles of this study, the effectiveness of communication process between the managers of the public health sector and OPAS website can help outlining the information system available in the HSD website.
\end{abstract}

\section{KEYWORDS}

Organizational communication. Public health managers. Information behavior patterns. Communication process. Information systems.

\section{INTRODUÇÃO}

Este artigo trata da relação entre padrões de comportamento informacional de usuários e delineamento de um sistema de informação da Organização Pan-Americana de Saúde/ Organização Mundial da Saúde (OPAS/OMS) no Brasil, fundamentado na abordagem dos processos de comunicação organizacional. Seu desenvolvimento objetivou investigar questões relacionadas a dois aspectos que fundamentaram teoricamente a investigação. $O$ primeiro visou à identificação dos padrões de comportamento informacional dos usuários do sistema estudado. O segundo buscou caracterizar o fluxo de informação entre seus principais atores.

O ambiente estudado foi o da Unidade Técnica de Desenvolvimento de Sistemas e Serviços de Saúde (HSD), da OPAS/OMS. A HSD está diretamente subordinada à Representação da OPAS no Brasil, tendo uma relação hierarquicamente equivalente às demais unidades daquela organização. No contexto da OPAS/OMS, a HSD é pioneira na implementação de iniciativas com o uso da Internet como instrumento de cooperação técnica (ICT).

O estudo adotou o conceito de sistema de atividade humana, em virtude de o problema tratado ocorrer em um ambiente organizacional, complexo por natureza, que envolve tanto sistemas de atividades realizadas na organização, quanto um sistema social composto das interações entre seus atores. A alternativa metodológica escolhida, portanto, foi a metodologia de sistemas flexíveis (MSF), que busca permitir a identificação de ações de melhoria em situações problemáticas investigadas, dentro do enfoque sistêmico (CHECKLAND, 1999; WILSON, 2000). São abordados no estudo aspectos do processo de comunicação no contexto organizacional, da informação e comportamento informacional de usuários, de sistemas de informação, tomada de decisão, tecnologia da informação e gestão da saúde pública no Brasil a partir da Constituição de 1988.

Os resultados do estudo fornecem subsídios para que o sistema de informação em questão possa ser melhorado a partir de uma proposta de mudanças que leva em conta os padrões de comportamento informacional de seus usuários na busca e uso das informações necessárias à consecução de suas atividades. 


\section{COMUNICAÇÃO ORGANIZACIONAL E COMPORTAMENTO INFORMACIONAL: ANÁLISE DA LITERATURA RELEVANTE}

$\mathrm{Na}$ discussão das questões identificadas como relevantes para o embasamento teórico desse artigo, destacam-se o processo de comunicação organizacional e os padrões de comportamento informacional de usuários de sistemas de informação. A discussão dessas questões se fundamentou na análise da literatura e permitiu tanto a construção do referencial teórico do estudo, quanto seu delineamento metodológico. Esta seção apresenta as discussões que fundamentam teoricamente o estudo, compreendendo questões sobre o processo de comunicação no contexto organizacional, comportamento informacional de usuários e sistemas de informação, tomada de decisão e tecnologias da informação.

\section{O processo de comunicação}

O processo de comunicação, neste estudo, é conformado por um organismo internacional, com o papel primordial de emissor, e por profissionais que atuam na gestão da saúde pública, no papel primordial de receptores. A compreensão de como se dá este processo tem relevância para a proposição de ações que possam torná-lo mais efetivo. Normalmente, para seu melhor entendimento, são utilizados modelos que, apesar de não conseguirem registrar a dinamicidade do processo de comunicação, permitem visualizar a relação entre os principais elementos que compõem, facilitando sua compreensão. $\mathrm{O}$ estudo da evolução dos modelos que ilustram o processo de comunicação permite identificar modelos que passam de lineares a circulares, helicoidais e na forma de espiral, todos apresentando elementos importantes a serem considerados quando de sua representação. A partir da consideração dos elementos apresentados por uma variedade de modelos, identificou-se o de Tubbs (TUBBS; MOSS, 2003) como o mais adequado para representar o processo de comunicação no contexto organizacional analisado. Dessa forma, o modelo do processo de comunicação proposto por Tubbs incorpora e/ou adapta elementos de modelos que o antecederam, propõe um aspecto dinâmico de representação e destaca o elemento insumo (estímulos), como pode ser observado na figura 1.

É importante destacar que a questão central deste estudo considera que a comunicação no contexto organizacional torna-se efetiva quando permite a troca de informações, e esta, a construção do conhecimento necessário à tomada de decisão. Nesse sentido, parece possível considerar que o processo de comunicação deve envolver, entre outras questões, os padrões de comportamento informacional de seus atores como elemento essencial para sua efetividade. Isso, porém, requer o aprofundamento dos aspectos relacionados à informação, sua necessidade, busca e uso e aos serviços e sistemas de informação.
No que concerne à informação, destaca-se o fato de que tem sido estudada dentro de uma variedade de campos do conhecimento. Por este motivo, sua conceituação é diversificada e complexa, o que aponta para a dificuldade de análise, a depender do enfoque que lhe é atribuído e de suas diferentes dimensões explicativas e conceituais (MORAES, 1998). Ou seja, a definição do que seja informação não é uma tarefa simples, não havendo para ela uma abordagem central no campo científico, nem mesmo no campo da ciência da informação. Esse panorama é refletido por meio de diversas definições e/ou interpretações do termo informação adotadas por estudiosos da área.

\section{Figura 1}

\section{Modelo da comunicação de Tubbs (TUBBS; MOSS, 2003)}

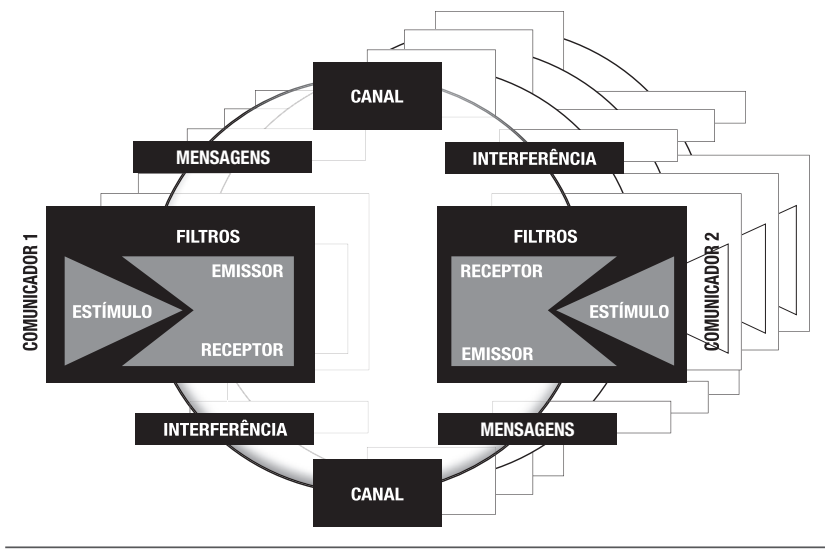

No presente estudo, a informação é considerada em termos de sua utilização por profissionais que atuam no contexto da saúde pública, representando a mensagem trocada entre emissores e receptores. É, pois, parte de um processo de comunicação que deve valorizar o receptor, preocupando-se com seu uso efetivo como etapa primordial para a ação.

A pertinência da informação no contexto da saúde pública relaciona-se ao modelo de gestão adotado por esse setor. Qualquer transformação das práticas sanitárias decorrentes de mudanças sociais, necessidades e problemas de saúde da população irá influenciar na forma como o gestor agirá para responder a essas questões. Dependerá, portanto, do tipo de informação a ser utilizada para dar subsídio a suas decisões e a suas ações (PAIM; ALMEIDA FILHO, 1998).

No Brasil, a partir da Constituição de 1988, a gestão da saúde passou por mudanças significativas nomeadas, em seu conjunto, como Reforma Sanitária Brasileira (CASTRO, 2002). Dessa forma, o novo perfil do gestor de saúde pública, delineado pela Reforma Sanitária, passou a demandar que os acervos informacionais de organizações do setor ofereçam informação técnica e científica direcionadas ao apoio à tomada de decisão em saúde pública. 
Esse cenário aponta para outra questão abordada no estudo aqui relatado. Trata-se da compreensão de como os usuários de acervos informacionais se comportam em relação à informação e como isso constitui uma etapa importante na proposta de melhoria de serviços de informação. A abordagem implica, assim, estudar o comportamento informacional humano dentro do paradigma dos estudos de usuários, incluindo aspectos que interferem na necessidade, busca e uso da informação.

\section{O comportamento informacional}

Um dos modelos propostos para estudar o comportamento informacional humano foi elaborado por Wilson (1981) e inclui os conceitos de necessidade, busca, transferência e uso de informação. Em relação a essa questão, ao descrever os fatores que influenciam a necessidade e o comportamento de busca de informação, Wilson (1981) afirma que existem, além das concepções de necessidade afetivas, cognitivas e psicológicas, aquelas relacionadas ao ambiente em que o indivíduo está inserido. É este o aspecto que fundamenta a discussão a respeito do comportamento informacional no presente estudo.

Wilson (1981) explica que o comportamento informacional humano ocorre com base no seguinte: o indivíduo, ao perceber sua necessidade de informação, engaja-se em um comportamento de busca de informação. Na busca, ele pode utilizar os sistemas ou outros canais formais de acesso à informação e até mesmo trocar informações de modo informal, com outras pessoas. Tendo sucesso na busca, usa a informação que pode satisfazer a sua necessidade, além de poder transferila a outras pessoas.

Neste estudo, os padrões de comportamento informacional de usuários de sistema de informação de organismo internacional da área de saúde são considerados parte importante no seu delineamento. São, também, tidos como parte do processo de comunicação, podendo, nesse sentido, influenciar a efetividade tanto do sistema de informação quanto do processo de comunicação. Isso, por sua vez, depende do quanto essas questões correspondem à situação do usuário e de quanto o usuário potencialmente usa os serviços de informação (SONNENWALD; IIVONEN, 1999). A identificação cuidadosa da real necessidade de informação do usuário é, portanto, base essencial para o planejamento, implementação e operacionalização de sistemas de informação (DEVADASON; PATRAP LINGAN, 1996), discutidas sucintamente a seguir.

\section{Sistemas de informação, tomada de decisão e tecnologia da informação}

Os sistemas de informação (SI) são tratados neste estudo sob o aspecto de sua relevância para a tomada de decisão (TD) e sua relação com a tecnologia, como o canal por meio do qual emissor e receptor trocam mensagens dentro de um processo de comunicação. Para Adeoti-Adekeye (1997), qualquer definição de sistemas de informação deve considerar pessoas, tecnologia e processos que facilitam a geração, o uso e a transferência da informação.

A provisão da informação por um sistema de informação deve permitir dar suporte à ação a ser realizada, utilizando tecnologia apropriada para apoiar seus usuários na seleção, ampliação, rejeição, atenuação ou distorção das informações recebidas, de acordo com suas próprias percepções. Isso permite que o conhecimento seja criado e, a partir dele, o estabelecimento de propostas de ação e tomada de decisão (CHECKLAND; HOLWELL, 1998). Considera-se neste estudo que, no contexto da saúde pública brasileira, o sistema de informação da HSD/OPAS pode desempenhar tarefa fundamental no processo de gestão em saúde, à medida que ofereça subsídios para a tomada de decisão.

A tomada de decisão constitui uma atividade importante para as ações governamentais no contexto da saúde pública. Pode, destarte, ser entendida como o filtro que ajuda a direcionar a troca de mensagens entre emissor e receptores. A informação técnica ou científica utilizada e processada pelos profissionais que atuam na gestão da saúde pública no Brasil subsidia o planejamento das ações. Em contrapartida, o impacto resultante dessas ações em saúde deve refletir os objetivos nacionais do setor e permitir que gestores sejam beneficiados pela sua utilização. Para que isso aconteça, deve-se considerar a utilização da tecnologia da informação como instrumento que potencializa o acesso à informação de qualidade.

A tecnologia da informação tem primordial papel como base para sistemas de informação, por trazer a possibilidade do aumento no acesso e na qualidade da informação. Nos ambientes organizacionais, a tecnologia desempenha papel fundamental, compreendendo o conjunto de conhecimentos, sistemas e equipamentos utilizados para tratamento, organização e disseminação de informações necessárias à tomada de decisão (TAKAHASHI, 2000).

Na área da saúde pública, a crescente produção de informação técnica e científica, especificamente na atividade de gestão, trouxe a necessidade de adotar um recurso que facilitasse e agilizasse a transmissão da informação a ser usada na tomada de decisão. Com este propósito, número variado de instituições tem tornado disponíveis informações voltadas para a atividade de gestão no setor. Dentre elas, citam-se o Ministério da Saúde, as secretarias estaduais e municipais de saúde, as instituições de ensino e de pesquisa e os organismos internacionais.

Neste estudo, portanto, o Sistema de Informação da HSD/ OPAS é considerado um instrumento de apoio à tomada de decisão. Agregam-se a essa questão as abordagens do processo de comunicação e dos padrões de comportamento 
informacional. O relacionamento entre esses três tópicos, portanto, conformam a abordagem teórica discutida a partir da literatura considerada relevante e que forneceu a base para montar a fundamentação teórica e os procedimentos metodológicos do estudo.

Nesse contexto, o problema no estudo foi tratado sob dois enfoques. O primeiro, epistemológico, diz respeito ao processo de comunicação no contexto organizacional e o papel que sistemas de informação, apoiados por tecnologias da informação e comunicação, exercem na gestão da informação (criação, disseminação e uso), levando em conta o comportamento informacional de seus usuários. O segundo, metodológico, relaciona-se com a contribuição da abordagem sistêmica e da fenomenologia, mais especificamente o conceito de sistema de atividade humana, com base na metodologia de sistemas flexíveis, de Peter Checkland, e no método da pesquisa-ação.

\section{Referencial teórico da pesquisa}

A construção do referencial teórico do estudo fundamentase no relacionamento entre o processo de comunicação no contexto organizacional, o comportamento de necessidade, busca e uso de informação por usuários de um sistema de informação apoiado em tecnologia, e na própria noção de sistemas de informação. Baseado nos modelos conceituais de Checkland e Howell (sistemas de informação), Tubbs (processo de comunicação) e Wilson (comportamento informacional de usuários), o estudo se fundamenta na premissa de que a efetividade do processo de comunicação entre um sistema de informação e seus usuários, no contexto organizacional, depende da correspondência próxima entre os estímulos (insumos) dos emissores e receptores da comunicação.

É importante notar que as necessidades de informação dos gestores são influenciadas pela função que exercem, a qual, por sua vez, sofre influência do contexto "gestão de saúde pública no Brasil”. É, portanto, pressuposto básico do presente estudo que o aperfeiçoamento de sistemas de informação, com base nos padrões de comportamento informacional de seus usuários, pode melhorar o processo de comunicação no ambiente organizacional. Assim, a relação entre os principais elementos do processo de comunicação, as questões que refletem o comportamento informacional de seus atores, usuários do sistema que compõem o processo, e as informações geradas e emitidas pelo sistema de informação apoiado por tecnologias de informação e comunicação configura o modelo que serve de embasamento teórico do presente estudo, como ilustrado na figura 2.

Fenomenologia, abordagem sistêmica e metodologia de sistemas flexíveis

Para estudar possíveis melhorias no sistema de informação da HSD/OPaS a partir de uma proposta de mudanças que leve em conta os padrões de comportamento informacional de seus usuários na busca e uso das informações necessárias à consecução de suas atividades, foi utilizada a metodologia de sistemas flexíveis (MSF). Tomou-se como base a abordagem fenomenológica, buscando identificar, dentro do ambiente organizacional em questão, aspectos relevantes do contexto, a partir da perspectiva de cada ator e sua estrutura subjetiva de significados (MAYRING, 2002).

A aplicação da MSF buscou, assim, incorporar a visão dos vários atores envolvidos no processo estudado, explicitando a situação para a qual serão propostas melhorias (COSTA, 2003). A abordagem do problema fundamenta-se no pensamento sistêmico, na medida em que a metodologia a ser aplicada no contexto organizacional relaciona-se diretamente com o conceito de sistemas.

\section{Figura 2}

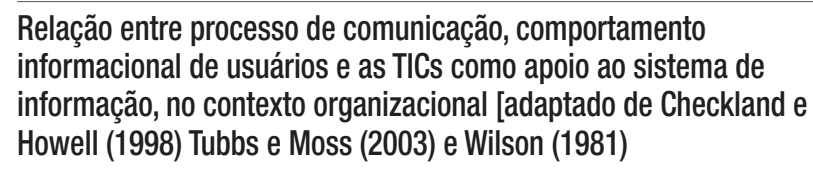

Howell (1998) Tubbs e Moss (2003) e Wilson (1981)

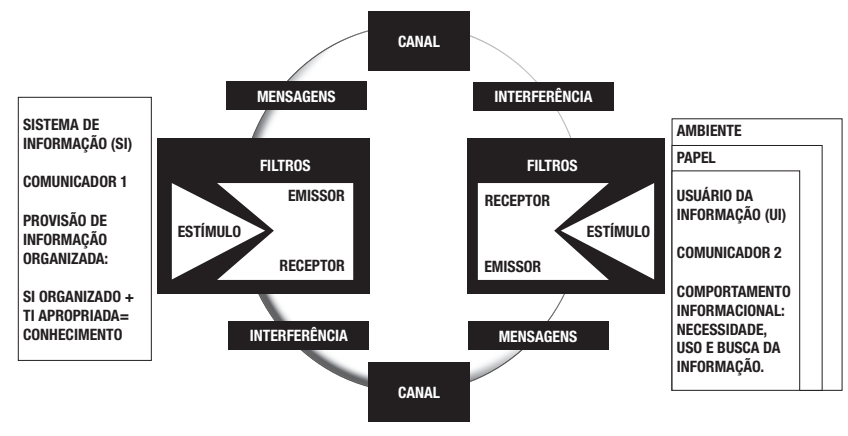

A MSF consiste em sete estágios de análise (figura 3), sendo expressa em forma de diagrama no qual se notam dois tipos de atividade: as que se inserem no mundo real e as que representam o pensamento sistêmico a respeito do que se observa no mundo real. As questões representadas na figura 2 e o norteamento obtido por meio da MSF permitiram definir os procedimentos metodológicos adequados ao estudo.

\section{Figura 3}

Estágios da MSF (Fonte: Checkland, 1999)

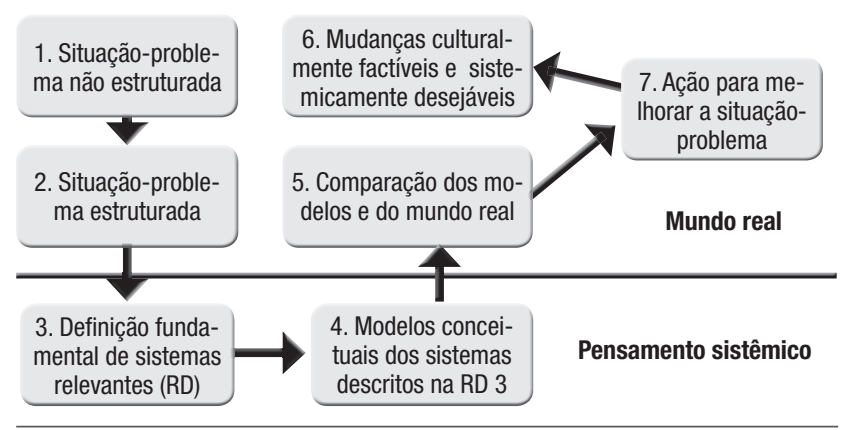




\section{PROCEDIMENTOS METODOLÓGICOS}

A metodologia de sistemas flexíveis foi aplicada na situação considerada problemática do ambiente da OPAS/OMS, mais especificamente, na Unidade Técnica de Desenvolvimento de Sistemas e Serviços de Saúde (HSD). Foram coletados dados que permitissem analisar a efetividade do processo de comunicação, considerando a informação tornada disponível pelo sítio Web HSD aos profissionais que atuam na área de gestão da saúde pública.

A coleta de dados consistiu de análise documental, entrevista e questionário. Os dados da pesquisa foram coletados e analisados no período de novembro de 2005 a fevereiro de 2006.

O quadro 1 apresenta o universo total da gestão da saúde pública no Brasil e a amostra definida para o estudo.

\section{Quadro 1}

Universo total da gestão da saúde pública no Brasil e amostra do estudo

\begin{tabular}{|c|c|c|c|}
\hline \multirow{3}{*}{$\begin{array}{l}\text { Universo total da } \\
\text { gestão da saúde } \\
\text { pública no Brasil }\end{array}$} & Gestores municipais & \multicolumn{2}{|c|}{5.563} \\
\hline & Gestores estaduais & \multicolumn{2}{|c|}{27} \\
\hline & Ministro da saúde & \multicolumn{2}{|c|}{1} \\
\hline \multirow{4}{*}{$\begin{array}{l}\text { Amostra não } \\
\text { probabilística } \\
\text { intencional }\end{array}$} & $\begin{array}{l}\text { Profissionais da OPAS que se } \\
\text { relacionam diretamente com } 0 \\
\text { sítio web HSD }\end{array}$ & \multicolumn{2}{|c|}{4} \\
\hline & $\begin{array}{l}\text { Profissionais que usam/conhecem } \\
\text { o sítio web HSD }\end{array}$ & \multicolumn{2}{|c|}{5} \\
\hline & $\begin{array}{l}\text { Novos profissionais da área } \\
\text { de gestão da saúde pública } \\
\text { cadastrados no sítio web da HSD, } \\
\text { de janeiro a dezembro de } 2005 \text {. }\end{array}$ & 157 & \multirow{2}{*}{$\begin{array}{l}\text { Total: } \\
620\end{array}$} \\
\hline & $\begin{array}{c}\text { Gestores estaduais e municipais } \\
\text { da saúde que possuem correio } \\
\text { eletrônico disponível por seus } \\
\text { respectivos conselhos }\end{array}$ & 473 & \\
\hline
\end{tabular}

Todos os profissionais que se relacionam diretamente com o sítio Web (quatro, lotados na unidade técnica) foram incluídos nessa amostra. Do total dos que usam/conhecem o sítio Web HSD, foram incluídos cinco, selecionados por nível de gestão (federal, estadual e municipal) e por região brasileira: um federal (CO), dois estaduais (CO e S) e dois municipais (NE e SE). A coleta de dados nesses dois grupos se deu por meio de entrevistas.

Novos profissionais e gestores foram abordados por meio de questionário, totalizando 630 questionários, encaminhados por meio de aplicativo de acesso livre pela Internet (www. surveymonkey.com). Foram selecionados todos os novos profissionais (157) cujo e-mail institucional foi cadastrado no sítio Web entre janeiro e dezembro de 2005 (indicativo de maior interesse no uso da internet). No extrato dos gestores, foram incluídos todos os 27 estaduais e todos os municipais que possuíam e-mail atualizado na mala direta do Conselho Nacional dos Secretários Municipais de Saúde (446). Do total de questionários usados para coleta de dados (620), foram obtidas 50,16\% de respostas (316 questionários) até a datalimite estabelecida para coleta.

\section{ANÁLISE DOS DADOS}

A análise dos dados do estudo é apresentada com base nos sete estágios da MSF, tal como descritos na figura 3.

\section{Situação-problema não estruturada (estágio 1 da MSF)}

Para caracterizar o processo de comunicação entre o sítio Web da HSD e seus usuários, foram analisados documentos que permitiram identificar o momento político da definição de iniciativas que visam ao uso da Internet como instrumento de Cooperação Técnica da HSD. Assim, foram consultados os documentos que descrevem a missão e a visão da OPAS, os principais eixos de cooperação técnica da HSD e o documentofonte da iniciativa de utilização da Internet como instrumento de cooperação técnica (ICT). Complementando a análise documental, foram analisados os dados coletados por meio dos cadastros no sítio HSD.

Os resultados dessa análise apontaram para o fato de que a informação tornada disponível pelo sistema de informação da HSD possui grande capilaridade, tanto no setor acadêmico quanto na área de gestão da saúde. Pode-se perceber que a ICT, realizada por meio da Web da HSD, alcançou um nível considerável de acessos (uma média de 1.000 acessos diários, conforme dados obtidos do aplicativo AWStats). Contudo, não se pode comprovar se esta informação está sendo utilizada de acordo com a finalidade própria dessa cooperação.

Dados complementares foram também coletados a partir de entrevistas realizadas junto a quatro profissionais que atuam diretamente nessa atividade e cinco profissionais da gestão da saúde pública que usam e/ou conhecem o sítio Web HSD. Adicionalmente, aos profissionais que atuam na gestão da saúde pública e aos profissionais da gestão estadual e municipal, definidos na amostra, foi aplicado um questionário via correio eletrônico. Os dados analisados permitiram identificar as principais questões relacionadas ao seu perfil de comportamento informacional, propiciando o que a MSF define como "estruturação da situação-problema" investigada, a qual foi analisada no estágio 2 da metodologia.

\section{Situação-problema estruturada (estágio 2 da MSF)}

Os resultados obtidos por meio da análise documental, das entrevistas e dos questionários forneceram a base de informações necessária ao entendimento e estruturação da 
situação problemática investigada. Foram identificados os elementos da estrutura, dos processos e do meio ambiente dentro do contexto do estudo, bem como as relações entre esses elementos, a fim de que as características do sistema de informação, do comportamento informacional de seus usuários e do processo de comunicação permitissem estruturar a situação-problema. A figura 4 ilustra o processo de comunicação entre a HSD e profissionais que atuam na gestão da saúde pública, adaptado do modelo de comunicação de Tubbs (TUBBS; MOSS, 2003), e norteia a elaboração da rich picture (RP) no sentido de apontar as questões relevantes a serem consideradas para a proposição de ações que melhorem o processo de comunicação estudado.

Como se pode observar, o processo de comunicação organizacional adaptado e considerado mais adequado apresenta como comunicador 1 a HSD, a qual emite mensagens para os profissionais da área de gestão da saúde pública e da academia, entre outros, cadastrados ou não em sua web, via meio eletrônico. A informação previamente analisada pela HSD passa por um filtro baseado no conhecimento do comunicador 1 a respeito da missão/visão da Organização, de sua vivência na área e do perfil/ambiente de trabalho do comunicador 2. Algumas interferências notadas nesta primeira parte do fluxo da informação são problemas tecnológicos e a forma como este conteúdo na web será divulgado. Os comunicadores 2 filtram a informação tornada disponível pela página de acordo com seu interesse. Supondo que a informação seja acessada, sua utilização poderá gerar a tomada de decisão, o que poderá retornar à HSD por canal formal, sob a interferência dos meios eletrônicos, caracterizando o feedback do processo.

Figura 4

Modelo da comunicação organizacional da HSD, baseado em Tubbs e Moss (2003)

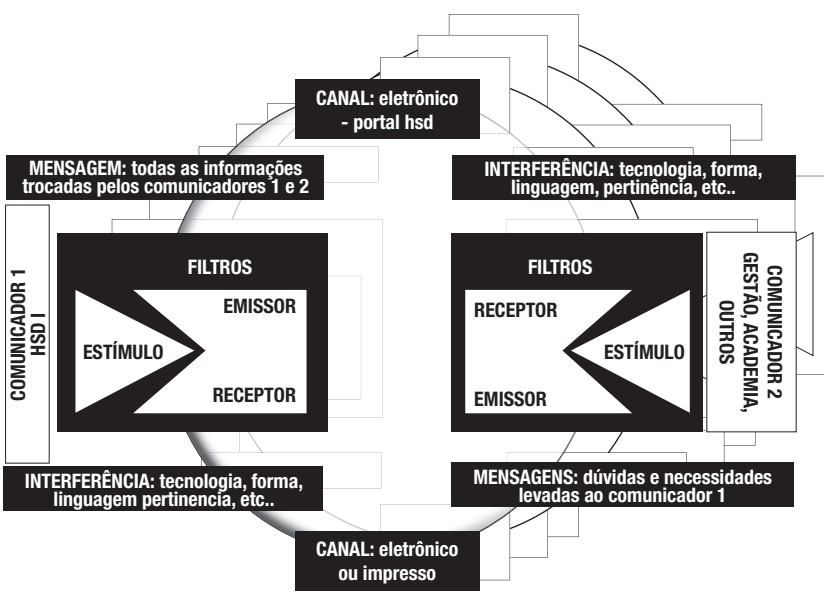

Além de ter sido possível visualizar como se dá o processo de comunicação no contexto estudado, foi possível analisar questões relevantes para nortear a proposição de um sistema de atividade humana capaz de melhorar a situação-problema investigada. Dessa forma, a rich picture elaborada para o estudo representa o processo de comunicação entre a OPAS e os profissionais que atuam na área de gestão da saúde pública (figura 5). Mais que isso, permitiu apontar as questões relevantes a serem levadas em conta na fase seguinte, de definição de sistemas relevantes (root definitions).

\section{Definições fundamentais de sistemas relevantes: Root definitions - RD (estágio 3 da MSF)}

Na elaboração da definição fundamental (root definition - RD), buscou-se capturar a essência do sistema identificado como mais relevante, a partir das questões relevantes retratadas na rich picture. $\mathrm{O}$ sistema proposto incorpora, portanto, as atividades consideradas significativas para seu desempenho, destacando os principais elementos que devem constar de uma RD bem formulada. Isso, por sua vez, foi possível a partir da definição dos elementos do CATWOE (espécie de check list para definição de uma RD bem elaborada), como mostrado a seguir.

\section{CATWOE}

\begin{tabular}{l|l}
\hline \multirow{4}{*}{ C } & CLIENTES DO SISTEMA PROPOSTO \\
- Usuários do sítio web HSD com e-mails correspondentes \\
a instituições de gestão da saúde pública, cadastrados no \\
período de janeiro a dezembro de 2005. \\
- Profissionais que atuam na gestão da saúde pública, nas \\
três esferas de governo, e que possuem cadastro atualizado \\
de e-mail.
\end{tabular}


Figura 5

\section{Rich picture da situação-problema estruturada}
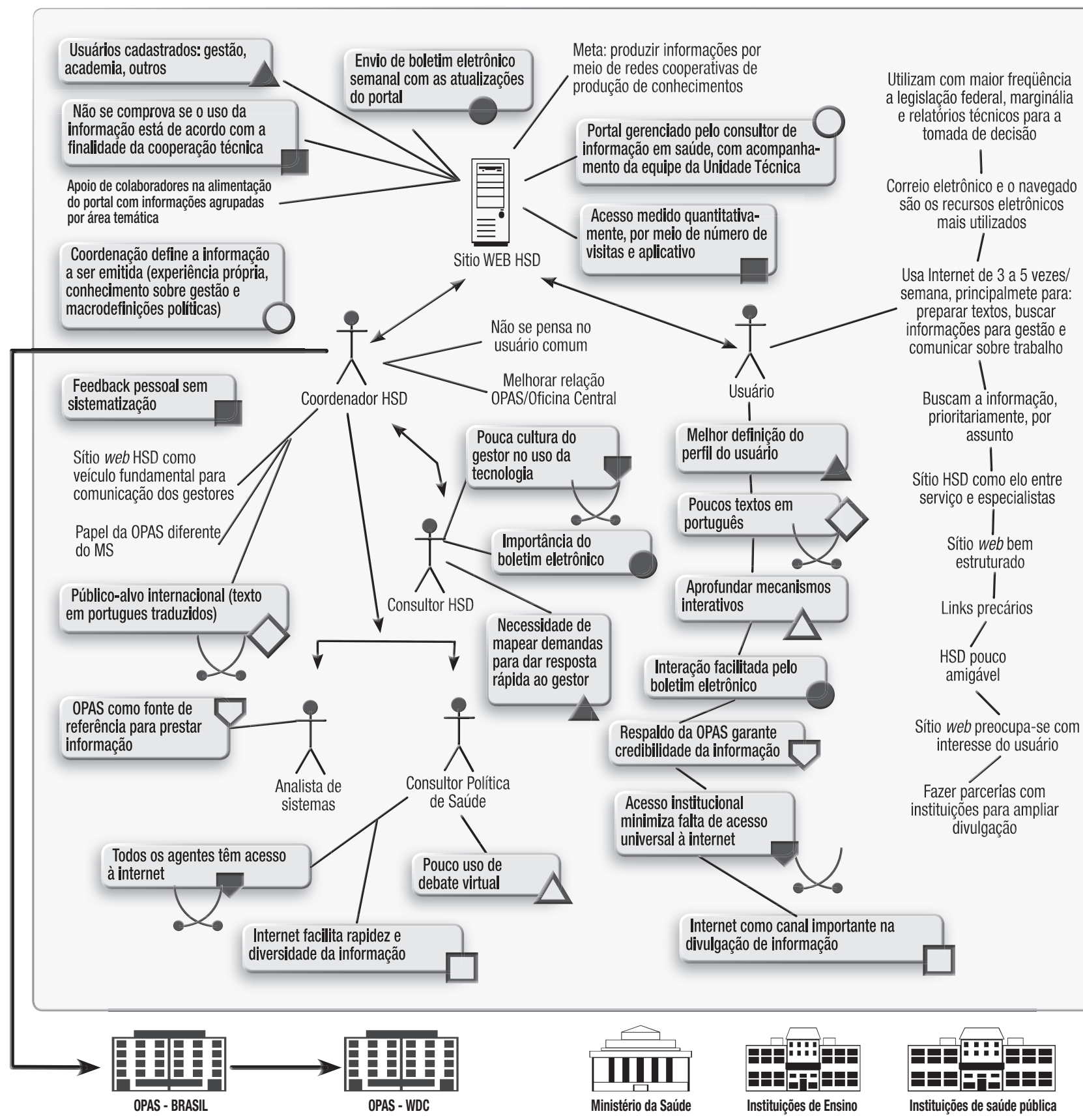

LEGENDA GRÁFICO

Conflito

Responsável pelo gerenciamento do sítio web

Importância do boletim eletônico

Uso da tecnologia

para prestar informação

Comentários sobre Internet

Feedback
Público-alvo

Aprofundar mecanismos interativos

Definição do idioma dos

textos a serem disseminados 
$\mathrm{RD}$ do sistema de comunicação organizacional proposto

Sistema de atividade humana de propriedade da HSD que transforma a comunicação organizacional, da qual fazem parte os profissionais que atuam diretamente junto ao sítio web HSD e os profissionais da área de gestão da saúde pública nas três esferas de governo - atualmente sem mecanismos que permitam identificar como estabelecer uma comunicação organizacional efetiva e alcançar os objetivos já traçados pela iniciativa de uso da Internet como instrumento de cooperação técnica, por meio do sítio web HSD - em um sistema que promova uma comunicação organizacional efetiva no sentido de prover informação que corresponda ao perfil de necessidades informacionais de seus usuários. A provisão de informação por meio de um sistema baseado em tecnologia terá seu escopo limitado ao sítio web da HSD devido às restrições impostas pela estrutura de comunicação organizacional da OPAS como um todo e das suas contrapartes nacionais.

Tal como estabelece a MSF, foi definido o modelo conceitual (estágio 4 da metodologia), derivado da RD elaborada. $\mathrm{O}$ objetivo do modelo é identificar e relacionar, logicamente, as ações necessárias à efetiva transformação proposta, tal como descrito a seguir.

\section{Modelo conceitual (estágio 4 da MSF)}

A elaboração do modelo conceitual do presente estudo baseouse no processo de transformação definido na $\mathrm{RD}$. A partir desse processo, foram definidas as ações mínimas necessárias para que a transformação ocorra, de fato. Cada uma das ações foi disposta em uma ordem cronológica e ilustrada em um diagrama representando o modelo conceitual (figura 6). Dentro da abordagem iterativa da MSF, o modelo conceitual embasa a elaboração de uma agenda de comparação que, conforme o resultado obtido, permite retornar a um ou mais dos estágios anteriores, se necessário, reiniciando a análise. É importante ressaltar que as ações constantes do Modelo Conceitual constituem, na orientação de Checkland, o mínimo necessário para que a transformação seja realizada. Mínimo necessário no sentido em que as ações são apresentadas de modo abrangente. É possível, portanto, que uma ou mais ações sejam, no futuro, transformadas em subsistema, dependendo de sua complexidade.

Figura 6

Modelo conceitual do processo de comunicação organizacional entre sítio web HSD e usuários
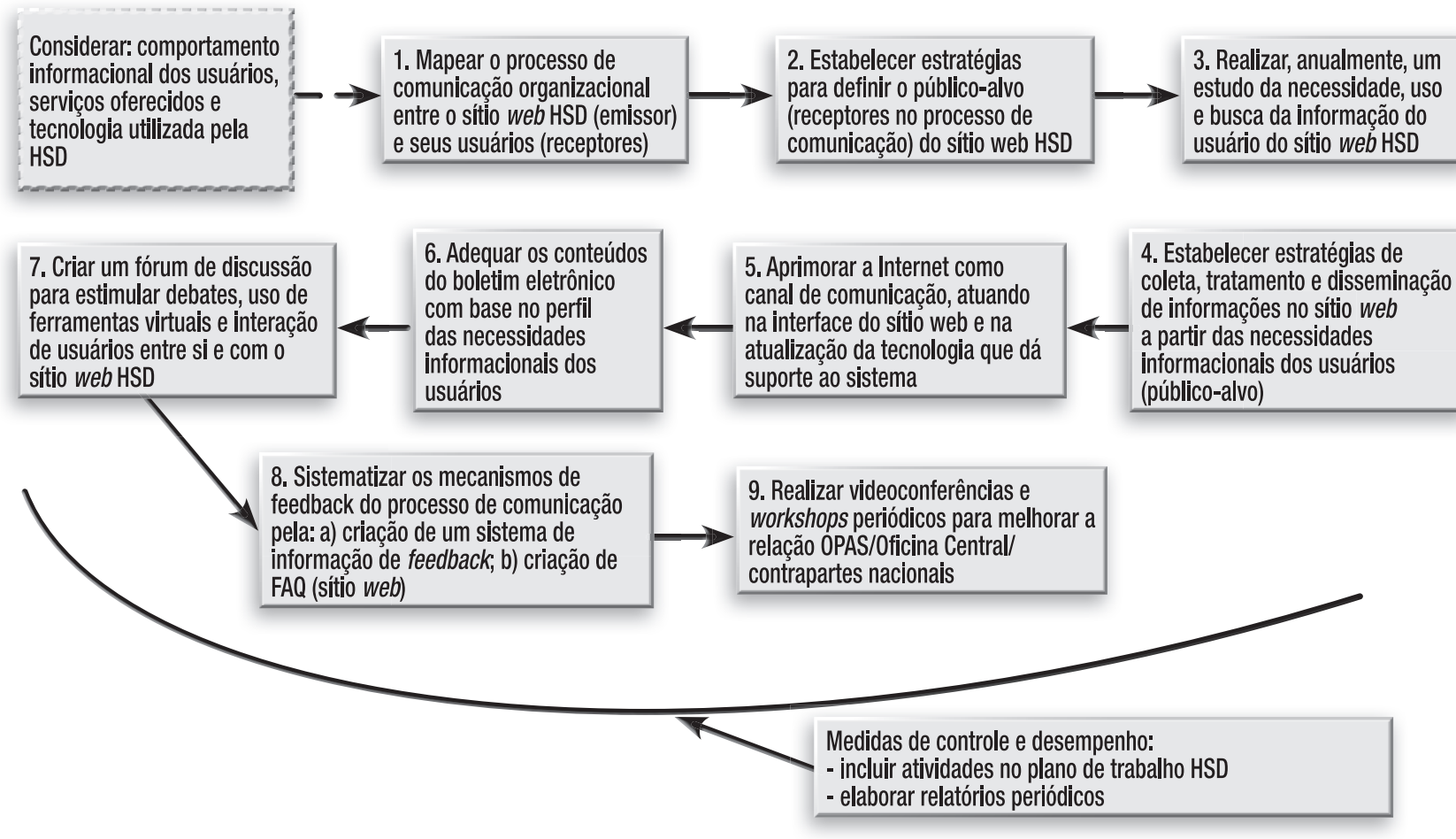


\section{Comparação (estágio 5 da MSF)}

A partir das ações propostas no modelo conceitual, é possível pensar em uma agenda que permita voltar aos sujeitos cujas percepções e visões nortearam a definição do sistema de atividade humana proposto. É preciso, também, avaliar se são culturalmente viáveis e sistemicamente desejáveis. Por culturalmente viáveis entende-se a avaliação com base em valores, crenças, regras e padrões, entre outros aspectos culturais compartilhados no ambiente da HSD e que, levados em conta, viabilizam sua implementação. Por sistemicamente desejáveis levam-se em conta as ações que devem, efetivamente, constituir os elementos do sistema proposto.
Neste estágio da metodologia, utilizou-se o método da discussão geral, conforme descrito por Wilson (1990). Por meio desse método, confrontam-se aspectos da realidade atual (ilustrada na rich picture) com as ações no modelo conceitual. Dessa comparação, derivam-se possíveis mudanças, a partir da avaliação dos sujeitos envolvidos na situação-problema. $\mathrm{Na}$ consecução dessa agenda, buscou-se um debate sobre "o que" existe e "como" existe, a fim de que a mudança possa acontecer. O quadro 2 sintetiza o resultado desse estágio da MSF.

Definição de mudanças desejáveis e viáveis (estágio 6 da MSF)

A partir dos resultados do estágio anterior, examinaram-se as mudanças propostas, definidas como desejáveis e viáveis, as

\section{Quadro 2}

Resultado do estágio 5 (Agenda de comparação entre as ações do sistema proposto e a situação problema estruturada no estágio 2)

\begin{tabular}{|c|c|c|c|c|c|c|c|c|}
\hline \multirow{4}{*}{ Ações propostas } & \multicolumn{8}{|c|}{ Critérios de julgamento } \\
\hline & \multirow{3}{*}{$\begin{array}{l}\text { Existe na } \\
\text { situação } \\
\text { real? }\end{array}$} & \multirow{2}{*}{\multicolumn{4}{|c|}{$\begin{array}{l}\text { SE SIM: Como julga sua } \\
\text { realização? }\end{array}$}} & \multicolumn{2}{|c|}{ SE NÃO: } & \multirow{3}{*}{$\begin{array}{l}\text { A ação deve ser } \\
\text { implementada? }\end{array}$} \\
\hline & & & & & & \multirow{2}{*}{$\begin{array}{c}\text { A ação proposta } \\
\text { é culturalmente } \\
\text { viável? * }\end{array}$} & \multirow{2}{*}{$\begin{array}{c}\text { É } \\
\text { sistemicamente } \\
\text { desejável? }{ }^{\star \star}\end{array}$} & \\
\hline & & Ruim & Regular & Bom & Excelente & & & \\
\hline $\begin{array}{l}\text { 1. Mapear o processo de comunicação } \\
\text { organizacional entre o sítio web HSD } \\
\text { (emissor) e seus usuários(receptores) }\end{array}$ & NÃO (3) & & & & & $\operatorname{SIM}(3)$ & $\operatorname{SIM}(3)$ & $\operatorname{SIM}(3)$ \\
\hline $\begin{array}{l}\text { 2. Estabelecer estratégias para definir o } \\
\text { público-alvo (receptores no processo de } \\
\text { comunicação) do sítio web HSD }\end{array}$ & NÃO (3) & & & & & $\operatorname{SIM}(3)$ & $\operatorname{SIM}(3)$ & $\operatorname{SIM}(3)$ \\
\hline $\begin{array}{l}\text { 3. Realizar, anualmente, um estudo da } \\
\text { necessidade, uso e busca da informação } \\
\text { do usuário do sítio web HSD }\end{array}$ & $\begin{array}{l}\text { NÃO (2) } \\
\text { SIM (1) }\end{array}$ & 1 & & & & $\operatorname{SIM}(2)$ & $\operatorname{SIM}(2)$ & $\operatorname{SIM}(2)$ \\
\hline $\begin{array}{l}\text { 4. Estabelecer estratégias de coleta, } \\
\text { tratamento e disseminação de } \\
\text { informações no sítio web a partir das } \\
\text { necessidades informacionais dos usuários } \\
\text { (público-alvo) }\end{array}$ & $\begin{array}{l}\text { NÃO (2) } \\
\text { SIM (1) }\end{array}$ & & & 1 & & $\operatorname{SIM}(2)$ & $\operatorname{SIM}(2)$ & $\operatorname{SIM}(2)$ \\
\hline $\begin{array}{l}\text { 5. Aprimorar a Internet como canal de } \\
\text { comunicação, atuando na interface do } \\
\text { sítio web e na atualização da tecnologia } \\
\text { que dá suporte ao sistema }\end{array}$ & $\begin{array}{l}\text { SIM (2) } \\
\text { NÃO (1) }\end{array}$ & & 1 & 1 & & $\operatorname{SIM}(1)$ & $\operatorname{SIM}(1)$ & $\operatorname{SIM}(1)$ \\
\hline $\begin{array}{l}\text { 6. Adequar os conteúdos do boletim } \\
\text { eletrônico com base no perfil das } \\
\text { necessidades informacionais dos usuários }\end{array}$ & NÃO (3) & & & & & $\operatorname{SIM}(3)$ & $\operatorname{SIM}(3)$ & $\operatorname{SIM}(3)$ \\
\hline $\begin{array}{l}\text { 7. Criar um fórum de discussão para } \\
\text { estimular debates, uso de ferramentas } \\
\text { virtuais e interação de usuários entre si e } \\
\text { com o sítio web HSD }\end{array}$ & NÃO (3) & & & & & $\operatorname{SIM}(3)$ & $\begin{array}{l}\operatorname{SIM}(2) \\
\text { NÃO (1) }\end{array}$ & $\begin{array}{l}\operatorname{SIM}(2) \\
\operatorname{NÃO}(1)\end{array}$ \\
\hline $\begin{array}{l}\text { 8. Sistematizar os mecanismos de feedback } \\
\text { do processo de comunicação pela: a) } \\
\text { criação de } 1 \text { sistema de informação de } \\
\text { feedback; b) criaçãa de FAQ (sítio web) }\end{array}$ & NÃO (3) & & & & & $\operatorname{SIM}(3)$ & $\operatorname{SIM}(3)$ & $\begin{array}{l}\operatorname{SIM}(2) \\
\operatorname{NA\tilde {0}}(1)\end{array}$ \\
\hline $\begin{array}{l}\text { 9. Realizar videoconferências e workshops } \\
\text { periódicos para melhorar a relação OPAS/ } \\
\text { Oficina Central/contrapartes nacionais }\end{array}$ & NÃO (3) & & & & & $\begin{array}{l}\text { NÃO (2) } \\
\text { SIM (1) }\end{array}$ & $\begin{array}{l}\text { NÃO (2) } \\
\text { SIM (1) }\end{array}$ & $\begin{array}{l}\text { NÃO (2) } \\
\text { SIM (1) }\end{array}$ \\
\hline
\end{tabular}

* Do ponto de vista da CULTURA (valores, crenças, normas etc) da HSD/OPAS, o Sr. (a) considera possível mudar / criar a atividade? ** 0 Sr. (a) considera possivel que a atividade constitua um elemento do sistema que transforma a comunicação em que está inserida a HSD/OPAS do status atual para o proposto? Fonte: Madeira (2005) 
quais devem ser incluídas em um plano de ação. Para seguir ao estágio de implementação dessas ações, é necessário discutir, em relação a cada uma das propostas, a técnica a ser utilizada, os recursos necessários e a duração, entre outros aspectos. Devido ao tempo de elaboração deste estudo, não foi possível aprofundar estes aspectos do plano de ação, nem avançar no estágio seguinte, o que sugere a necessidade de retomar estas questões em estudos futuros.

\section{Resultados da análise dos dados}

Pelos resultados apresentados, as melhorias no processo de comunicação de que fazem parte a HSD, por meio de seu sistema de informação (sítio Web), e os profissionais que atuam na gestão da saúde pública parecem estar relacionadas à inclusão dos padrões de comportamento informacional dos usuários como insumo (input) para as definições e decisões relacionadas ao desenho do sistema de informações. Ou seja, há uma relação entre processo de comunicação, comportamento informacional de usuários e as TICs como apoio ao sistema de informação, no contexto da HSD/OPAS, conforme descrito na figura adaptada de Checkland e Howell (1998), Tubbs e Moss (2003) e Wilson (1981).

Nesse sentido, parece possível identificar uma relação possível entre padrões de comportamento informacional (WILSON, 1981) e comunicação efetiva (TUBBS; MOSS, 2003), no contexto que envolve o uso de sistemas de informação (CHECKLAND; HOWELL, 1998). Essa noção, no presente estudo, ficou evidenciada pelo reconhecimento da importância dos padrões de comportamento informacional como insumo à comunicação.

Sumariando os resultados obtidos, a identificação dos perfis de necessidade dos usuários do sítio Web HSD em relação ao uso de tecnologias da informação, como a Internet, mostrou que:

- a maioria dos profissionais é do sexo feminino, de idade entre 41 e 50 anos, com maior nível de formação em licenciatura/bacharelado, desempenhando suas atividades em gestão da saúde pública há, em média, 10 anos;

- prevalece a utilização da Internet com uma freqüência alta, principalmente para atividades relacionadas à preparação de textos (relatórios, artigos etc.) e busca de informações para subsidiar a gestão e comunicar-se sobre assunto de trabalho;

- os computadores utilizados para consecução das atividades dos profissionais está situado tanto no trabalho quanto em casa, sendo que o correio eletrônico e o navegador são os recursos eletrônicos mais utilizados, podendo-se considerar também como relevantes o uso de motores de busca e editores de texto;

- as fontes preferencialmente utilizadas pelos profissionais são bases de dados científicas, as estatísticas, os sítios de instituições públicas nacionais ou internacionais e de instituições de pesquisa e ensino, as publicações impressas ou os colegas, sendo possível detectar maior tendência ao uso de sítios de instituições públicas nacionais;

- maior freqüência de uso da legislação federal, marginália e relatórios técnicos como tipos de informação para a tomada de decisão, embora artigos de periódico científico e estatísticas também sejam bastante consultados;

c há uma produção mensal de relatos de experiências, relatórios técnicos, memorandos internos e documentos oficiais, não sendo freqüente a produção de artigos;

- a busca da informação dá-se, prioritariamente, por assunto, tanto no ambiente da Internet quanto no sítio Web HSD, sendo que o comportamento informacional na busca de informação por meio de outros pontos de acesso, como autores, tipo de arquivo, título e editor, não varia muito, com um ligeiro aumento do idioma em relação ao sítio Web HSD;

- o boletim eletrônico é utilizado como uma ferramenta que facilita a interação entre o sítio web HSD e seus usuários; - a informação disponibilizada pela OPAS é considerada de relevância e credibilidade na área da gestão da saúde pública;

- a Internet é considerada como um canal que amplia a capilaridade da informação.

Verificou-se, ainda, que o delineamento dos serviços oferecidos pelo sitio Web HSD revela uma evolução de seu sistema de informação. Destaca-se a capilaridade da informação disponível no sítio Web HSD, tanto no setor acadêmico quanto na área de gestão da saúde, o que aponta para o fato de que a ICT tem dado resultado, ainda que não seja possível comprovar se esta informação está sendo utilizada de acordo com a finalidade da cooperação.

Também notaram-se tanto semelhanças quanto discrepância entre os padrões de comportamento informacional de usuários do sítio web da HSD e os serviços de informação por ele oferecidos. Destacam-se como aspectos semelhantes a utilização de sítios de instituições públicas internacionais, a utilização de relatórios técnicos produzidos por instituições públicas, a Internet como canal de divulgação da informação, a credibilidade dada à informação disseminada pela OPAS e a importância do boletim eletrônico na disseminação da informação. Como discrepância, destaca-se a necessidade de melhor definição do perfil do usuário.

\section{CONCLUSÃO}

Dando destaque à questão da efetividade do processo de comunicação organizacional e aos elementos que o compõem, o presente estudo tomou por base um sistema de informação baseado em tecnologia apropriada que leva em conta as necessidades, busca e uso de informação por 
parte de seus usuários. Portanto, a efetividade do processo de comunicação entre HSD/OMS e seus usuários dependerá de o seu sistema de informação (sítio web) estar baseado no padrão de comportamento informacional dos profissionais de gestão da saúde pública e prover, pelo uso de tecnologia adequada, informação relevante para a tomada de decisão.

Com base nesse referencial e em relação aos objetivos propostos para o estudo, concluiu-se que:

A partir da adaptação do modelo de Tubbs (TUBBS; MOSS, 2003), foi possível caracterizar o processo de comunicação entre a HSD e os profissionais da gestão de saúde pública, identificando-se os principais elementos do processo.

Foi possível definir um conjunto de ações necessárias à efetividade do processo de comunicação entre a HSD/OPAS e os profissionais da gestão de saúde pública no Brasil, quais sejam:

- mapeamento do processo de comunicação organizacional entre o sítio web HSD (emissor) e seus usuários (receptores) para melhor visualização do processo, o que permite uma oferta de informação mais adequada aos seus usuários, desde que o SI considere o comportamento informacional dos receptores;

- estabelecimento de estratégias para definir o públicoalvo (receptores no processo de comunicação) do sítio web HSD para delineamento do sistema a partir de seus padrões de comportamento;

- realização anual de estudos da necessidade, uso e busca da informação do usuário do sítio web, visando $a$ tornar atualizado o conhecimento sobre o perfil do comportamento informacional dos usuários;

- estabelecimento de estratégias de coleta, tratamento e disseminação de informações no sítio web a partir das necessidades informacionais dos usuários (público-alvo), com o objetivo de garantir que a informação tornada disponível possa contribuir no processo de tomada de decisão pelos profissionais da saúde pública;

c aprimoramento da Internet como canal de comunicação (interface mais amigável, melhor navegação e localização de documentos);

- adequação dos conteúdos do boletim eletrônico com base no perfil das necessidades informacionais dos usuários.

\section{CONSIDERAÇÕES FINAIS}

As ações constantes do delineamento proposto pelo estudo estão definidas em nível macro, sendo necessárias gestões que capacitem e conscientizem os técnicos da organização de seu papel primordial de emissores, dentro de um processo de comunicação, assim como possibilitar um entendimento do processo de comunicação em que estão inseridos na OPAS. Sugere-se que o estudo seja avaliado e sejam retomados os estágios necessários para a implementação de seus resultados, promovendo a reflexão dos atores que constituem o sistema de informação da HSD/OPAS sobre o processo de comunicação, levando-se em conta, portanto, a iteratividade própria da metodologia de sistemas flexíveis.

\section{Contribuições do estudo}

O contexto organizacional em que se desenvolveu o presente estudo permitiu identificar contribuições teóricas para a ciência da informação e práticas para a OPAS. Estas contribuições adicionam questões relevantes ao corpo de conhecimento da ciência da informação e ajudam na melhoria do sistema de informação da organização estudada, respectivamente.

\section{Considerações adicionais}

Durante o desenvolvimento deste estudo, foram observadas questões importantes que não fizeram parte do objetivo principal do trabalho. No entanto, decorreram deste, e podem trazer contribuições para estudos futuros. São lições aprendidas e sugestões para o aprofundamento de alguns temas.

\section{Sugestões para estudos futuros}

Este estudo aponta para a relevância de se considerar o padrão de comportamento informacional de usuários quando do delineamento de SI apoiados em tecnologia. Aponta, igualmente, para a necessidade de que esses padrões sejam levados em conta como insumo à emissão de mensagens entre os comunicadores (emissor e receptor), a fim de que o processo de comunicação no contexto organizacional se torne efetivo. Isso pode resultar no direcionamento do conhecimento útil à pessoa certa, em tempo hábil e de forma compreensível, causando impacto sobre Sistemas de Informaçãos e, em última análise, melhorando-os efetivamente. Assim, é importante ressaltar que o processo de comunicação torna-se efetivo quando se baseia em padrões de comportamento informacional de seus atores, o que propicia a melhoria de sistemas de informação.

Observe-se que essa constatação tem relação com a gestão do conhecimento, por levar em conta parte essencial da criação, coleta e disseminação do conhecimento para tornar a organização mais atuante. A comunicação, pois, deve ser tomada como um componente crucial da gestão do conhecimento, tal como afirma Teixeira Filho (2001), entre outros especialistas no tópico. A partir desse estudo, o aprofundamento das questões de comunicação e gestão do conhecimento no contexto organizacional mostra-se, definitivamente, um tópico importante para discussão, com grande aplicabilidade no desenvolvimento de trabalhos futuros.

Artigo recebido em 10/10/2007

e aceito para publicação em 16/05/2008 


\section{REFERÊNCIAS}

ADEOTI-ADEKEYE, W. B. The importance of management information systems. Library Review, v. 46, n. 5, 1997. Disponível em:

$<$ http: $/ /$ miranda.emeraldinsight $. \mathrm{com} / \mathrm{vl}=4296895 / \mathrm{cl}=59 / \mathrm{nw}=1 /$ $\mathrm{fm}=$ docpdf/rpsv/cw/mcb/00242535/v46n5/s2/p318 > . Acesso em: jun. 2005.

CASTRO, E. Informação para apoio à tomada de decisão em saúde: parâmetros de produção de informação territorializada. 2002. 79 f. Dissertação (Mestrado em Ciência da Comunicação)- Universidade de São Paulo, São Paulo, 2002.

CHECKLAND, P. B. Systems thinking, systems practice: includes a 30-year retrospective. Chichester: Wiley, 1999.

\section{Wiley, 1998.}

; HOLWELL, S. Information, systems and information systems. Lancaster:

COSTA, S. M. S. A metodologia de sistemas flexíveis aplicada a estudos em ciência da informação: uma experiência pedagógica. Transinformação, Campinas, v. 15, n. 2, 2003.

DEVADASON, F. J.; PATRAP LINGAM, P. A methodology for the identification of information needs of users. In: IFLA GENERAL CONFERENCE, 62., 1996, Beijing. Proceedings... The Hage: IFLA, 1996.

MADEIRA, F. A comunicação organizacional e científica no âmbito do CID: a aplicação da metodologia de sistemas flexíveis. 2005. Monografia (Graduação em biblioteconomia)- Departamento de Ciência da Informação, Universidade de Brasília, 2005.
MAYRING, P. Introdução à pesquisa social qualitativa: uma introdução para pensar qualitativamente. 5. ed. Weinheim: Beltz, 2002.

MORAES, I. H. S. Informações em saúde: para andarilhos e argonautas de uma tecnodemocracia emancipadora. 1998. 274 f. Tese (Doutorado em saúde pública)-Escola Nacional de Saúde Pública, Fundação Oswaldo Cruz, Rio de Janeiro, 1998.

PAIM, J. S.; ALMEIDA FILHO, N. Saúde coletiva: uma nova saúde pública ou campo aberto a novos paradigmas. Revista de Saúde Pública, São Paulo, v. 32, n. 4, p. 299-316, ago. 1998.

SONNENWALD, D.; IIVONEN, M. An integrated human information behavior research framework for information studies. Library $\mathbb{E}$ Information Science Research, Chapel Hill, v. 21, n. 4, p. 429-457, Nov. 1999.

TAKAHASHI, T. (Org.). Sociedade da informação no Brasil: livro verde. Brasília: Ministério da Ciência e Tecnologia, 2000. Disponível em: $<$ http://diamante.socinfo. org.br/livro_verde/index.htm>. Acesso em: jan. 2006.

TUBBS, S. L.; MOSS, S. Human communication: principles and contexts. 9. ed. Boston: McGraw-Hill, 2003.

WILSON, B. Systems: concepts, methodologies and applications. 2nd ed. Chichester: Wiley, 1990.

WILSON, T. D. On user studies and information needs. Joumal of Documentation, v. 37, n. 1, 1981. 